\title{
Independence of solution sets and minimal asymptotic bases
}

\author{
by
}

Paul Erdős (Budapest), Melvyn B. Nathanson (Bronx, N.Y.) and Prasad Tetali (Murray Hill, N.J.)

1. Introduction. Let $A$ be a set of positive integers, and let $k \geq 2$ be a fixed integer. Let $r_{A}(n)$ denote the number of representations of $n$ in the form

$$
n=a_{1}+a_{2}+\ldots+a_{k},
$$

where

$$
0<a_{1} \leq a_{2} \leq \ldots \leq a_{k}
$$

and $a_{i} \in A$ for $i=1, \ldots, k$. Let $r_{A}^{\prime}(n)$ denote the number of "strict" representations of $n$ in the form

$$
n=a_{1}+a_{2}+\ldots+a_{k},
$$

where

$$
0<a_{1}<a_{2}<\ldots<a_{k}
$$

and $a_{i} \in A$ for $i=1, \ldots, k$. The set $A$ is called an asymptotic basis of order $k$ if there exists a natural number $n_{1}$ such that $r_{A}(n)>0$ for all $n \geq n_{1}$. The set $A$ is called a strict asymptotic basis of order $k$ if there exists a natural number $n_{1}$ such that $r_{A}^{\prime}(n)>0$ for all $n \geq n_{1}$. All bases considered in this paper will be either asymptotic or strict asymptotic bases of order $k$. Erdős and Tetali [7] gave a probabilistic construction of a strict asymptotic basis $\mathcal{S}$ of order $k$ whose representation function satisfies $\log n \ll r_{\mathcal{S}}^{\prime}(n) \ll \log n$.

An asymptotic basis (resp. strict asymptotic basis) $A$ is called minimal if the removal of any element from the basis destroys all representations of some infinite sequence of numbers, that is, $A \backslash\{a\}$ is not an asymptotic

Research of the second author supported in part by grants from the PSC-CUNY Research Award Program, was done while visiting the Center for Discrete Mathematics and Theoretical Computer Science (DIMACS), Rutgers University, Piscataway, NJ 08855. 
basis (resp. strict asymptotic basis) for any $a \in A$. An asymptotic basis (resp. strict asymptotic basis) $A$ is defined to be $\aleph_{0}$-minimal if $A \backslash F$ is an asymptotic basis (resp. strict asymptotic basis) for every finite subset $F$ of $A$, but $A \backslash I$ fails to be a basis for every infinite subset $I$ of $A$. Erdös and Nathanson $[3,4]$ survey results concerning minimal asymptotic bases. In [2], they derived conditions under which an asymptotic basis of order 2 contains a minimal asymptotic basis, and they also constructed in [1] a family of $\aleph_{0}$-minimal asymptotic bases of order 2 .

This paper has two aims. First, we give a simple set of criteria under which an asymptotic basis (resp. strict asymptotic basis) contains a minimal asymptotic basis (resp. strict asymptotic basis). These criteria also enable us to construct $\aleph_{0}$-minimal bases. Second, we show that the strict asymptotic basis $\mathcal{S}$ constructed in [7] satisfies this set of criteria and so contains a minimal as well as an $\aleph_{0}$-minimal asymptotic basis. These results answer two important questions posed in [4].

Notation. Let $k A$ denote the set of all sums of $k$ elements of $A$, and let $k^{\wedge} A$ denote the set of all sums of $k$ distinct elements of $A$. Let $r_{A}(n ; a)$ (resp. $\left.r_{A}^{\prime}(n ; a)\right)$ denote the number of representations of $n$ in the form (1)-(2) (resp. (3)-(4)) such that $a_{i}=a$ for some $i=1, \ldots, k$. The solution set of $n$, denoted by $S_{A}(n)$ (resp. $S_{A}^{\prime}(n)$ ), is the set of integers in $A$ that appear in some representation of $n$; that is,

$$
S_{A}(n)=\left\{a \in A \mid r_{A}(n ; a)>0\right\}
$$

and

$$
S_{A}^{\prime}(n)=\left\{a \in A \mid r_{A}^{\prime}(n ; a)>0\right\} .
$$

2. Minimal and $\aleph_{0}$-minimal asymptotic bases. Erdôs and Nathanson [2] discovered a set of simple criteria for an asymptotic basis of order 2 to contain a minimal asymptotic basis of order 2 . We shall generalize this result to asymptotic bases of order $k \geq 3$. The following theorem is a natural extension of Theorem 3 of [2]. Condition (ii) is trivially satisfied in the case $k=2$, but is a nontrivial restriction for asymptotic bases of orders $k \geq 3$.

THEOREM 1. Let $A$ be a strictly increasing sequence of positive integers, and let $k \geq 2$. If

(i) $\lim _{n \rightarrow \infty} r_{A}(n)=\infty$,

(ii) $r_{A}(n ; a)$ is bounded for all $n \geq 1, a \in A$,

(iii) $\left|S_{A}(m) \cap S_{A}(n)\right|$ is bounded for all $m \neq n$,

then $A$ contains

(a) a minimal asymptotic basis of order $k$, and

(b) an $\aleph_{0}$-minimal asymptotic basis of order $k$. 
Proof. Let $r_{A}(n ; a) \leq c$ for all $n \geq 1$ and $a \in A$. It follows that if $F$ is any finite subset of $A$ and if $\left|F \cap S_{A}(n)\right| \leq w$, then

$$
r_{A \backslash F}(n) \geq r_{A}(n)-c w
$$

for all $n$, since the removal of any one element of $S_{A}(n)$ destroys at most $c$ representations of $n$. Let $\left|S_{A}(m) \cap S_{A}(n)\right| \leq d$ for all $m \neq n$. If $F \subseteq S_{A}(m)$, then

$$
\left|F \cap S_{A}(n)\right| \leq\left|S_{A}(m) \cap S_{A}(n)\right| \leq d,
$$

and so

$$
r_{A \backslash F}(n) \geq r_{A}(n)-c d
$$

for all $n \neq m$.

We shall use induction to construct a decreasing sequence of sets

$$
A=A_{0} \supseteq A_{1} \supseteq A_{2} \supseteq \ldots
$$

such that

$$
A^{*}=\bigcap_{j=0}^{\infty} A_{j}
$$

is a minimal asymptotic basis of order $k$. We shall also construct a second decreasing sequence of subsets of $A$ whose intersection is an $\aleph_{0}$-minimal asymptotic basis of order $k$.

Let $A_{0}=A$. Since $\lim _{n \rightarrow \infty} r_{A}(n)=\infty$, we can choose an integer $n_{1}$ so that $r_{A_{0}}(n)=r_{A}(n)>c(1+d)$ for all $n \geq n_{1}$.

Choose $a_{1}, b_{1} \in A_{0}$ such that $a_{1} \leq n_{1}$ and $b_{1}>k n_{1}$. Let $m_{1}=a_{1}+$ $(k-1) b_{1}$. Then

$$
k n_{1}<b_{1} \leq(k-1) b_{1}<m_{1}<k b_{1} .
$$

We shall construct a set $A_{1} \subseteq A_{0}$ such that $r_{A_{1}}(n)>0$ for all $n \geq n_{1}$, but $m_{1} \notin k\left(A_{1} \backslash\left\{a_{1}\right\}\right)$. Thus, every representation of $m_{1}$ as a sum of $k$ elements of $A_{1}$ must include the integer $a_{1}$ as a summand.

We first determine a subset $F_{1}$ of $A_{0}$ that "destroys" every representation of $m_{1}$ that does not include $a_{1}$ as a summand. Every such representation is of the form

$$
m_{1}=a_{1}^{\prime}+a_{2}^{\prime}+\ldots+a_{t}^{\prime}+(k-t) b_{1},
$$

where $a_{i}^{\prime} \in A_{0}$ and $a_{i}^{\prime} \neq a_{1}, b_{1}$ for $i=1,2, \ldots, t$. Note that $m_{1}<k b_{1}$ implies that $t \neq 0$. If $t=1$, then

$$
a_{1}+(k-1) b_{1}=m_{1}=a_{1}^{\prime}+(k-1) b_{1}
$$

implies that $a_{1}^{\prime}=a_{1}$, which is false. Therefore, $2 \leq t \leq k$. Let $a_{1}^{\prime} \leq a_{2}^{\prime} \leq$ $\ldots \leq a_{t}^{\prime}$. Then

$$
(k-1) b_{1}<m_{1} \leq t a_{t}^{\prime}+(k-t) b_{1} \leq k a_{t}^{\prime}+(k-2) b_{1}
$$


implies that

$$
a_{t}^{\prime}>b_{1} / k>n_{1} .
$$

Let $F_{1}$ be the set of all such integers $a_{t}^{\prime}$, and let $A_{1}=A_{0} \backslash F_{1}$. Then $F_{1} \subseteq\left[n_{1}+1, m_{1}\right]$. Since $a_{1}, b_{1} \notin F_{1}$, it follows that $m_{1}=a_{1}+(k-1) b_{1}$ is a representation of $m_{1}$ as a sum of $k$ elements of $A_{1}$, and so $r_{A_{1}}\left(m_{1}\right)>0$. On the other hand, we have destroyed every representation of $m_{1}$ as the sum of $k$ elements of $A_{0}$ all different from $a_{1}$, and so $m_{1} \notin k\left(A_{0} \backslash\left\{a_{1}\right\}\right)$.

Let $n \geq n_{1}, n \neq m_{1}$. Since $F_{1} \subseteq S_{A}\left(m_{1}\right)$, it follows that

$$
r_{A_{1}}(n)=r_{A \backslash F_{1}}(n) \geq r_{A}(n)-c d>c(1+d)-c d=c>0 .
$$

This completes the first step of the induction.

Let $j \geq 2$. Suppose we have constructed sets $A=A_{0} \supseteq A_{1} \supseteq \ldots \supseteq A_{j-1}$ and integers $n_{i}, a_{i}, m_{i}$ for $i=1, \ldots, j-1$ with the following properties:

(i) $k n_{1}<m_{1}<n_{2}<k n_{2}<m_{2}<n_{3}<\ldots<k n_{j-1}<m_{j-1}$,

(ii) $F_{i}=A_{i-1} \backslash A_{i} \subseteq\left[n_{i}+1, m_{i}\right]$ for $i=1, \ldots, j-1$,

(iii) $a_{1}, \ldots, a_{j-1} \in A_{j-1}$,

(iv) $r_{A_{j-1}}(n)>0$ for $n \geq n_{1}$,

(v) $m_{i} \notin k\left(A_{i} \backslash\left\{a_{i}\right\}\right)$ for $i=1, \ldots, j-1$.

We now construct the set $A_{j}$ and integers $n_{j}, a_{j}$, and $m_{j}$.

Let $G_{j}=A \backslash A_{j-1} \subseteq\left[1, m_{j-1}\right]$. Choose $n_{j}>m_{j-1}$ such that $r_{A}(n)>$ $c\left(j+d+\left|G_{j}\right|\right)$ for all $n \geq n_{j}$. Choose $a_{j}, b_{j} \in A_{j-1}$ such that $a_{j}<n_{j}$ and $b_{j}>k n_{j}$. Let $m_{j}=a_{j}+(k-1) b_{j}$. Then

$$
k n_{j}<b_{j} \leq(k-1) b_{j}<m_{j}<k b_{j} .
$$

Exactly as in the first step of the induction, we shall determine a subset $F_{j}$ of $A_{j-1}$ that "destroys" every representation of $m_{j}$ as a sum of $k$ elements of $A_{j-1}$ that does not include $a_{j}$ as a summand. Every such representation is of the form

$$
m_{j}=a_{1}^{\prime}+a_{2}^{\prime}+\ldots+a_{t}^{\prime}+(k-t) b_{j},
$$

where $2 \leq t \leq k$, and $a_{i}^{\prime} \in A_{j-1}, a_{i}^{\prime} \neq a_{j}, b_{j}$ for $i=1,2, \ldots, t$. Let

$$
a_{1}^{\prime} \leq a_{2}^{\prime} \leq \ldots \leq a_{t}^{\prime} \text {. }
$$

Then

$$
(k-1) b_{j}<m_{j} \leq t a_{t}^{\prime}+(k-t) b_{j} \leq k a_{t}^{\prime}+(k-2) b_{j}
$$

implies that

$$
a_{t}^{\prime}>b_{j} / k>n_{j} .
$$

Let $F_{j}$ be the set of all such integers $a_{t}^{\prime}$, and let $A_{j}=A_{j-1} \backslash F_{j}$. Then

$$
F_{j} \subseteq\left[n_{j}+1, m_{j}\right] \cap S_{A_{j-1}}\left(m_{j}\right) \subseteq\left[n_{j}+1, m_{j}\right] \cap S_{A}\left(m_{j}\right) .
$$

Since $a_{j}, b_{j} \notin F_{j}$, it follows that $m_{j}=a_{j}+(k-1) b_{j}$ is a representation of $m_{j}$ as a sum of $k$ elements of $A_{j}$, and so $r_{A_{j}}\left(m_{j}\right)>0$. However, $m_{j} \notin$ 
$k\left(A_{j} \backslash\left\{a_{j}\right\}\right)$, since the set $A_{j}$ was constructed so that every representation of $m_{j}$ as the sum of $k$ elements of $A_{j}$ has at least one summand equal to $a_{j}$.

Let $n_{1} \leq n \leq n_{j}$. Since $A_{j-1} \backslash A_{j}=F_{j} \subseteq\left[n_{j}+1, m_{j}\right]$, it follows that $r_{A_{j}}(n)=r_{A_{j-1}}(n)>0$. Let $n>n_{j}, n \neq m_{j}$. Since

$$
A \backslash A_{j}=F_{j} \cup G_{j}
$$

and

$$
\left(F_{j} \cup G_{j}\right) \cap S_{A}(n) \subseteq\left(F_{j} \cap S_{A}(n)\right) \cup G_{j} \subseteq\left(S_{A}\left(m_{j}\right) \cap S_{A}(n)\right) \cup G_{j},
$$

it follows that

$$
\left|\left(F_{j} \cup G_{j}\right) \cap S_{A}(n)\right| \leq d+\left|G_{j}\right|
$$

and so

$$
r_{A_{j}}(n) \geq r_{A}(n)-c\left(d+\left|G_{j}\right|\right)>c\left(j+d+\left|G_{j}\right|\right)-c\left(d+\left|G_{j}\right|\right)=c j>0 .
$$

This completes the induction.

Let $A^{*}=\bigcap_{j=1}^{\infty} A_{j}$. Let $n \geq n_{1}$. Choose $j \geq 1$ so that $n_{j} \leq n<n_{j+1}$. Since $A_{j} \backslash A^{*} \subseteq\left[n_{j+1}+1, \infty\right)$, it follows that

$$
r_{A^{*}}(n)=r_{A_{j}}(n)>c j>0,
$$

and so $A^{*}$ is an asymptotic basis of order $k$. Moreover, since

$$
m_{j} \notin k\left(A_{j} \backslash\left\{a_{j}\right\}\right)
$$

for every $j \geq 1$, it follows that

$$
m_{j} \notin k\left(A^{*} \backslash\left\{a_{j}\right\}\right) .
$$

Recall that at each step $j$ of the induction, we chose an integer $a_{j}$. We had complete freedom to select this integer, subject only to the conditions that $a_{j} \in A_{j-1}$ and $a_{j} \leq n_{j}$. Let us choose these integers in such a way that every element $a \in A^{*}$ is chosen infinitely often, that is, if $a \in A^{*}$, then $a=a_{j}$ for infinitely many $j$. Then the set $A^{*}$ will be a minimal asymptotic basis of order $k$, since the deletion of any element $a \in A^{*}$ will destroy all representations of infinitely many integers $m_{j}$.

To construct an $\aleph_{0}$-minimal asymptotic basis, we choose the numbers $a_{j}$ such that, if $a \in A^{*}$, then $a=a_{j}$ for exactly one integer $j$. If an infinite subset $I$ is deleted from $A^{*}$, then there are infinitely many integers of the form $m_{j}$ that cannot be written as the sum of $k$ terms of $A \backslash I$, and so $A \backslash I$ is not an asymptotic basis of order $k$.

Let $F$ be a finite subset of $A^{*}$, and let $|F|=f$. We shall show that $A^{*} \backslash F$ is an asymptotic basis of order $k$.

Since $a_{j} \in F$ for exactly $f$ indices $j$, and since $b_{j} \in F$ for at most $f$ indices $j$, it follows that $m_{j} \in k\left(A^{*} \backslash F\right)$ for all but at most $2 f$ numbers $m_{j}$. 
Let $n \geq n_{f}, n \neq m_{j}$ for all $j$. Choose $j$ such that $n_{j} \leq n<n_{j+1}$. Then $j \geq f$. Since

$$
r_{A^{*}}(n)=r_{A_{j}}(n)>c j>0,
$$

and since each element of $F$ destroys at most $c$ representations of $n$, it follows that

$$
r_{A^{*} \backslash F}(n)>c j-c f \geq 0 .
$$

Thus, $A^{*} \backslash F$ is an asymptotic basis of order $k$, and so $A^{*}$ is an $\aleph_{0}$-minimal asymptotic basis of order $k$.

THEOREM 2. Let $A$ be a strictly increasing sequence of positive integers, and let $k \geq 2$. If

(i) $\lim _{n \rightarrow \infty} r_{A}^{\prime}(n)=\infty$,

(ii) $r_{A}^{\prime}(n ; a)$ is bounded for all $n \geq 1, a \in A$,

(iii) $\left|S_{A}^{\prime}(m) \cap S_{A}^{\prime}(n)\right|$ is bounded for all $m \neq n$,

then A contains

(a) a minimal strict asymptotic basis of order $k$, and

(b) an $\aleph_{0}$-minimal strict asymptotic basis of order $k$.

Proof. Let $r_{A}^{\prime}(n ; a) \leq c$ for all $n \geq 1$ and $a \in A$. It follows that if $F$ is any finite subset of $A$ and if $\left|F \cap S_{A}^{\prime}(n)\right| \leq w$, then

$$
r_{A \backslash F}^{\prime}(n) \geq r_{A}^{\prime}(n)-c w
$$

for all $n$, since the removal of any one element of $S_{A}^{\prime}(n)$ destroys at most $c$ representations of $n$. Let $\left|S_{A}^{\prime}(m) \cap S_{A}^{\prime}(n)\right| \leq d$ for all $m \neq n$. If $F \subseteq S_{A}^{\prime}(m)$, then

and so

$$
\left|F \cap S_{A}^{\prime}(n)\right| \leq\left|S_{A}^{\prime}(m) \cap S_{A}^{\prime}(n)\right| \leq d,
$$

for all $n \neq m$.

$$
r_{A \backslash F}^{\prime}(n) \geq r_{A}^{\prime}(n)-c d
$$

We shall use induction to construct a decreasing sequence of sets $A=$ $A_{0} \supseteq A_{1} \supseteq A_{2} \supseteq \ldots$ such that $\widehat{A}=\bigcap_{j=0}^{\infty} A_{j}$ is a strict minimal asymptotic basis of order $k$. We shall also construct a second decreasing sequence of subsets of $A$ whose intersection is an $\aleph_{0}$-minimal strict asymptotic basis of order $k$.

Let $A_{0}=A$. Since $\lim _{n \rightarrow \infty} r_{A}^{\prime}(n)=\infty$, we can choose an integer $n_{1}$ so that $r_{A_{0}}^{\prime}(n)=r_{A}^{\prime}(n)>c(1+d)$ for all $n \geq n_{1}$. Choose $k$ integers $a_{1}, b_{1,1}, b_{1,2}, \ldots, b_{1, k-1} \in A_{0}$ such that

$$
a_{1} \leq n_{1}<k n_{1}<b_{1,1}<b_{1,2}<\ldots<b_{1, k-1} .
$$

Let

$$
m_{1}=a_{1}+b_{1,1}+b_{1,2}+\ldots+b_{1, k-1} .
$$


We shall construct a set $A_{1} \subseteq A_{0}$ such that $r_{A_{1}}^{\prime}(n)>0$ for all $n \geq n_{1}$, and with the additional property that every representation of $m_{1}$ as a sum of $k$ distinct elements of $A_{1}$ must include the integer $a_{1}$ as a summand.

We first determine a subset $F_{1}$ of $A_{0}$ that "destroys" every strict representation of $m_{1}$ that does not include $a_{1}$ as a summand. Every such representation is of the form

$$
m_{1}=a_{1}^{\prime}+a_{2}^{\prime}+\ldots+a_{t}^{\prime}+b_{1, u_{1}}+b_{1, u_{2}}+\ldots+b_{1, u_{k-t}},
$$

where $2 \leq t \leq k$, and $a_{i}^{\prime} \in A_{0}, a_{i}^{\prime} \neq a_{1}, b_{1, u}$ for $i=1,2, \ldots, t$ and $u=$ $1, \ldots, k-1$. Let $a_{1}^{\prime}<a_{2}^{\prime}<\ldots<a_{t}^{\prime}$. Since $(k-1)-(k-t)=t-1 \geq 1$ it follows that

$$
\begin{aligned}
\left(b_{1,1}+b_{1,2}+\ldots+b_{1, k-1}\right)-\left(b_{1, u_{1}}+b_{1, u_{2}}+\ldots+b_{1, u_{k-t}}\right) & \\
& =b_{1, v_{1}}+b_{1, v_{2}}+\ldots+b_{1, v_{t-1}} \geq b_{1,1} .
\end{aligned}
$$

Then

$$
\begin{aligned}
m_{1} & =a_{1}+b_{1,1}+b_{1,2}+\ldots+b_{1, k-1} \\
& =a_{1}^{\prime}+a_{2}^{\prime}+\ldots+a_{t}^{\prime}+b_{1, u_{1}}+b_{1, u_{2}}+\ldots+b_{1, u_{k-t}}
\end{aligned}
$$

implies that

$$
\begin{aligned}
k n_{1} & <b_{1,1}<a_{1}+b_{1,1} \leq a_{1}+b_{1, v_{1}}+b_{1, v_{2}}+\ldots+b_{1, v_{t-1}} \\
& =m_{1}-\left(b_{1, u_{1}}+b_{1, u_{2}}+\ldots+b_{1, u_{k-t}}\right)=a_{1}^{\prime}+a_{2}^{\prime}+\ldots+a_{t}^{\prime}<t a_{t}^{\prime} \leq k a_{t}^{\prime}
\end{aligned}
$$

and so

$$
a_{t}^{\prime}>n_{1} \text {. }
$$

Let $F_{1}$ be the set of all such integers $a_{t}^{\prime}$, and let $A_{1}=A_{0} \backslash F_{1}$. Then $F_{1} \subseteq\left[n_{1}+\right.$ $\left.1, m_{1}\right]$. Since $a_{1}, b_{1,1}, b_{1,2}, \ldots, b_{1, k-1} \notin F_{1}$, it follows that $m_{1}=a_{1}+b_{1,1}+$ $b_{1,2}+\ldots+b_{1, k-1}$ is a representation of $m_{1}$ as a sum of $k$ distinct elements of $A_{1}$, and so $r_{A_{1}}^{\prime}\left(m_{1}\right)>0$. On the other hand, we have destroyed every representation of $m_{1}$ as the sum of $k$ distinct elements of $A_{0}$ all different from $a_{1}$, and so $m_{1} \notin k\left(A_{0} \backslash\left\{a_{1}\right\}\right)$.

Let $n \geq n_{1}, n \neq m_{1}$. Since $F_{1} \subseteq S_{A}^{\prime}\left(m_{1}\right)$, it follows that

$$
r_{A_{1}}^{\prime}(n)=r_{A \backslash F_{1}}^{\prime}(n) \geq r_{A}^{\prime}(n)-c d>c(1+d)-c d=c>0 .
$$

This completes the first step of the induction.

Let $j \geq 2$. Suppose we have constructed sets $A=A_{0} \supseteq A_{1} \supseteq \ldots \supseteq A_{j-1}$ and integers $n_{i}, a_{i}, m_{i}$ for $i=1, \ldots, j-1$ with the following properties:

(i) $k n_{1}<m_{1}<n_{2}<k n_{2}<m_{2}<n_{3}<\ldots<k n_{j-1}<m_{j-1}$,

(ii) $F_{i}=A_{i-1} \backslash A_{i} \subseteq\left[n_{i}+1, m_{i}\right]$ for $i=1, \ldots, j-1$,

(iii) $a_{1}, \ldots, a_{j-1} \in A_{j-1}$,

(iv) $r_{A_{j-1}}^{\prime}(n)>0$ for $n \geq n_{1}$,

(v) $m_{i} \notin k\left(A_{i} \backslash\left\{a_{i}\right\}\right)$ for $i=1, \ldots, j-1$.

We now construct the set $A_{j}$ and integers $n_{j}, a_{j}$, and $m_{j}$. 
Let $G_{j}=A \backslash A_{j-1} \subseteq\left[1, m_{j-1}\right]$. Choose $n_{j}>m_{j-1}$ such that

$$
r_{A}^{\prime}(n)>c\left(j+d+\left|G_{j}\right|\right)
$$

for all $n \geq n_{j}$. Choose $a_{j}, b_{j, 1}, b_{j, 2}, \ldots, b_{j, k-1} \in A_{j-1}$ such that

$$
a_{j} \leq n_{j}<k n_{j}<b_{j, 1}<b_{j, 2}<\ldots<b_{j, k-1} \text {. }
$$

Let

$$
m_{j}=a_{j}+b_{j, 1}+b_{j, 2}+\ldots+b_{j, k-1} .
$$

Exactly as in the first step of the induction, we shall determine a subset $F_{j}$ of $A_{0}$ that "destroys" every representation of $m_{j}$ as a sum of $k$ distinct elements of $A_{j-1}$ that does not include $a_{j}$ as a summand.

Every such representation is of the form

$$
m_{j}=a_{1}^{\prime}+a_{2}^{\prime}+\ldots+a_{t}^{\prime}+b_{j, u_{1}}+b_{j, u_{2}}+\ldots+b_{j, u_{k-t}},
$$

where $2 \leq t \leq k$, and $a_{i}^{\prime} \in A_{0}, a_{i}^{\prime} \neq a_{j}, b_{j, u}$ for $i=1,2, \ldots, t$ and $u=$ $1,2, \ldots, k-1$. Let

$$
a_{1}^{\prime}<a_{2}^{\prime}<\ldots<a_{t}^{\prime} .
$$

Since $(k-1)-(k-t)=t-1 \geq 1$, it follows that

$$
\begin{aligned}
\left(b_{j, 1}+b_{j, 2}+\ldots+b_{j, k-1}\right)-\left(b_{j, u_{1}}\right. & \left.+b_{j, u_{2}}+\ldots+b_{j, u_{k-t}}\right) \\
& =b_{j, v_{1}}+b_{j, v_{2}}+\ldots+b_{j, v_{t-1}} \geq b_{j, 1} .
\end{aligned}
$$

Then

implies that

$$
\begin{aligned}
m_{j} & =a_{j}+b_{j, 1}+b_{j, 2}+\ldots+b_{j, k-1} \\
& =a_{1}^{\prime}+a_{2}^{\prime}+\ldots+a_{t}^{\prime}+b_{j, u_{1}}+b_{j, u_{2}}+\ldots+b_{j, u_{k-t}}
\end{aligned}
$$

$$
\begin{aligned}
k n_{j} & <b_{j, 1}<a_{j}+b_{j, 1} \leq a_{j}+b_{j, v_{1}}+b_{j, v_{2}}+\ldots+b_{j, v_{t-1}} \\
& =m_{j}-\left(b_{j, u_{1}}+b_{j, u_{2}}+\ldots+b_{j, u_{k-t}}\right)=a_{1}^{\prime}+a_{2}^{\prime}+\ldots+a_{t}^{\prime}<t a_{t}^{\prime} \leq k a_{t}^{\prime}
\end{aligned}
$$

and so

$$
a_{t}^{\prime}>n_{j}
$$

Let $F_{j}$ be the set of all such integers $a_{t}^{\prime}$, and let $A_{j}=A_{j-1} \backslash F_{j}$. Then $F_{j} \subseteq\left[n_{j}+1, m_{j}\right]$. Since $a_{j}, b_{j, 1}, b_{j, 2}, \ldots, b_{j, k-1} \notin F_{j}$, it follows that $m_{j}=$ $a_{j}+b_{j, 1}+b_{j, 2}+\ldots+b_{j, k-1}$ is a representation of $m_{j}$ as a sum of $k$ elements of $A_{j}$, and so $r_{A_{j}}^{\prime}\left(m_{j}\right)>0$. On the other hand, we have destroyed every representation of $m_{j}$ as the sum of $k$ distinct elements of $A_{j-1}$ all different from $a_{j}$, and so $m_{j} \notin k\left(A_{j-1} \backslash\left\{a_{j}\right\}\right)$. Let $n_{1} \leq n \leq n_{j}$. Since $A_{j-1} \backslash A_{j}=$ $F_{j} \subseteq\left[n_{j}+1, m_{j}\right] \cap S_{A}^{\prime}\left(m_{j}\right)$, it follows that $r_{A_{j}}^{\prime}(n)=r_{A_{j-1}}^{\prime}(n)>0$. Let $n>n_{j}, n \neq m_{j}$. Since

$$
A \backslash A_{j}=F_{j} \cup G_{j}
$$

and

$$
\left(F_{j} \cup G_{j}\right) \cap S_{A}^{\prime}(n) \subseteq\left(F_{j} \cap S_{A}^{\prime}(n)\right) \cup G_{j} \subseteq\left(S_{A}^{\prime}\left(m_{j}\right) \cap S_{A}^{\prime}(n)\right) \cup G_{j},
$$


it follows that

$$
\left|\left(F_{j} \cup G_{j}\right) \cap S_{A}^{\prime}(n)\right| \leq d+\left|G_{j}\right|
$$

and so

$$
r_{A_{j}}^{\prime}(n) \geq r_{A}^{\prime}(n)-c\left(d+\left|G_{j}\right|\right)>c\left(j+d+\left|G_{j}\right|\right)-c\left(d+\left|G_{j}\right|\right)=c j>0 .
$$

This completes the induction.

Let $\widehat{A}=\bigcap_{j=1}^{\infty} A_{j}$. Let $n \geq n_{1}$. Choose $j \geq 1$ so that $n_{j} \leq n<n_{j+1}$. Since $A_{j} \backslash \widehat{A} \subseteq\left[n_{j+1}+1, \infty\right)$, it follows that

$$
r_{\hat{A}}^{\prime}(n)=r_{A_{j}}^{\prime}(n)>c j>0,
$$

and so $\widehat{A}$ is a strict asymptotic basis of order $k$. Moreover, for every $j \geq 1$, since $m_{j} \notin k\left(A_{j} \backslash\left\{a_{j}\right\}\right)$, it follows that

$$
m_{j} \notin k\left(\widehat{A} \backslash\left\{a_{j}\right\}\right) .
$$

Recall that at each step $j$ of the induction, we chose an integer $a_{j}$. We had complete freedom to select this integer, subject only to the conditions that $a_{j} \in A_{j-1}$ and $a_{j} \leq n_{j}$. Let us choose these integers in such a way that every element $a \in \widehat{A}$ is chosen infinitely often, that is, if $a \in \widehat{A}$, then $a=a_{j}$ for infinitely many $j$. Then the set $\widehat{A}$ will be a minimal asymptotic basis of order $k$, since the deletion of any element $a$ will destroy all representations of infinitely many integers $m_{j}$.

To construct an $\aleph_{0}$-minimal strict asymptotic basis, we choose the numbers $a_{j}$ such that, if $a \in \widehat{A}$, then $a=a_{j}$ for exactly one integer $j$. If an infinite subset $I$ is deleted from $\widehat{A}$, then there is an infinite increasing sequence of integers of the form $m_{j}$ that cannot be written as the sum of $k$ terms of $\widehat{A} \backslash I$, and so $\widehat{A} \backslash I$ is not a strict asymptotic basis of order $k$.

Let $F$ be a finite subset of $\widehat{A}$, and let $|F|=f$. We shall show that $\widehat{A} \backslash F$ is a strict asymptotic basis of order $k$.

Since $a_{j} \in F$ for exactly $f$ indices $j$, and since $b_{j, u} \in F$ for at most $f$ double indices $(j, u)$, it follows that $m_{j} \in k(\widehat{A} \backslash F)$ for all but at most $2 f$ numbers $m_{j}$.

Let $n \geq n_{f}, n \neq m_{j}$ for all $j$. Choose $j$ such that $n_{j} \leq n<n_{j+1}$. Then $j \geq f$. Since

$$
r_{\hat{A}}^{\prime}(n)=r_{A_{j}}^{\prime}(n)>c j>0,
$$

and since each element of $F$ destroys at most $c$ representations of $n$, it follows that

$$
r_{\hat{A} \backslash F}^{\prime}(n)>c j-c f=c(j-f) \geq 0 .
$$

Thus, $\widehat{A} \backslash F$ is a strict asymptotic basis of order $k$, and so $\widehat{A}$ is an $\aleph_{0}$-minimal strict asymptotic basis of order $k$. 
3. Independence of solution sets. Let $\mathcal{S}$ be the asymptotic basis constructed in [7]. In this section we want to prove that $\mathcal{S}$ satisfies the conditions of Theorems 1 and 2. That is, we prove the following theorem.

TheOREM 3. The asymptotic basis $\mathcal{S}$ contains the following:

(a) a minimal asymptotic basis of order $k$,

(b) a minimal strict asymptotic basis of order $k$,

(c) an $\aleph_{0}$-minimal asymptotic basis of order $k$, and

(d) an $\aleph_{0}$-minimal strict asymptotic basis of order $k$.

Proof. In view of the previous section, it suffices to verify that $\mathcal{S}$ satisfies the hypotheses of Theorems 1 and 2. We first prove, in Lemma 1 below, that it suffices to verify that $\mathcal{S}$ satisfies the hypothesis of Theorem 2 . The first criterion of the hypothesis of Theorem 2 is satisfied by $\mathcal{S}$, since $r_{\mathcal{S}}^{\prime}(n)=\Theta(\log n)$, which is the main result of [7]. Lemmas 2 and 3 in the following show that the asymptotic basis $\mathcal{S}$ does in fact satisfy the rest of the hypothesis of Theorem 2. (In short, Lemmas 1-3 below constitute the proof of this theorem.)

Suppose that $\mathcal{S}$ satisfies the hypothesis of Theorem 2. The following argument shows that $\mathcal{S}$ satisfies the hypothesis of Theorem 1 as well.

LEMMA 1. $r_{\mathcal{S}}(n)-r_{\mathcal{S}}^{\prime}(n)<\infty$ for all $n$.

Proof. Consider the representations that contribute to $r_{\mathcal{S}}(n)$ but not to $r_{\mathcal{S}}^{\prime}(n)$. The number of distinct elements in each such representation of $n$ is at least one and at most $k-1$. Consider a representation of $n$ with $l$ distinct elements, where $1 \leq l \leq k-1$, i.e.

$$
n=a_{1}+\ldots+a_{l}+a_{l+1}+\ldots+a_{k}, \quad a_{i} \in \mathcal{S}, a_{1}<\ldots<a_{l} .
$$

We will be done by showing that there are only finitely many representations of this form for each $n$.

Consider $m=n-\left(a_{l+1}+\ldots+a_{k}\right)=a_{1}+\ldots+a_{l}$. Equivalently, we want to show for each $m$, the number of representations (denoted by $r_{l}^{\prime}(m)$ ) as a sum of $l$ distinct elements from $\mathcal{S}$ is bounded.

By Lemma 10 of [7], we know that the number of representations of $n$ as a sum of $l$ distinct elements is bounded for $l<k$. Hence the lemma.

With this lemma, for the rest of this section it suffices to consider only the distinct representations, and verify that $\mathcal{S}$ satisfies the hypothesis of Theorem 2. The second criterion in Theorem 2 asserts that the number of representations of $n$ that use $a$ be bounded, for every $n \in \mathbb{N}$, and $a \in \mathcal{S}$.

Lemma 2. $r_{\mathcal{S}}^{\prime}(n ; a)$ is bounded for all $a \in \mathcal{S}$. 
Proof. Note that $r_{\mathcal{S}}^{\prime}(n ; a)=$ the number of representations (in $\mathcal{S}$ ) of $n-a$ as a sum of $k-1$ terms. Once again this follows from Lemma 10 of [7].

Finally, the following lemma proves that $\mathcal{S}$ meets the third criterion in Theorem 2.

Lemma 3. $\left|S_{\mathcal{S}}^{\prime}(m) \cap S_{\mathcal{S}}^{\prime}(n)\right|$ is bounded for all $m<n$.

Before we prove Lemma 3, we need a couple of technical lemmas. The idea is going to be similar to that of the proof of Lemma 10 of [7]; we first estimate the expected such number, and then bound the disjoint occurrences.

Let $R_{l}(n, m)$ represent the number of representations of $n$ and $m$ that overlap in $l$ numbers. (Note that $l \in[1, k-1]$.) Further, let $R_{l}^{*}(n, m)$ represent a maximal collection of "disjoint overlaps" - each overlapping pair of representations for $n$ and $m$ is disjoint from the other overlapping pairs. Also, let $R(n, m)$ and $R^{*}(n, m)$ denote the corresponding terms when no restriction is made on the size $(l)$ of the overlap.

Lemma 4. $E[R(n, m)] \leq n^{-l /(2 k)+o(1)}$.

Pr o of. Without loss of generality, let $m<n$. Then, for fixed $n$ and $m$, a typical overlapping pair of representations is of the following form:

$z_{1}+\ldots+z_{l}+x_{1}+\ldots+x_{k-l}=n, \quad z_{1}+\ldots+z_{l}+y_{1}+\ldots+y_{k-l}=m$,

where

$$
z_{1}+\ldots+z_{l}=t, \quad 1 \leq t<m
$$

Thus the expected value of $R_{l}(n, m)$ equals

$$
\begin{aligned}
\sum_{1 \leq t<m} \sum_{\begin{array}{c}
z_{1}+\ldots+z_{l}=t \\
x_{1}+\ldots+x_{k-l}=n-t \\
y_{1}+\ldots+y_{k-l}=m-t
\end{array}} & \operatorname{Pr}\left[z_{1}\right] \ldots \operatorname{Pr}\left[z_{l}\right] \\
& \times\left(\operatorname{Pr}\left[x_{1}\right] \ldots \operatorname{Pr}\left[x_{k-l}\right]\right)\left(\operatorname{Pr}\left[y_{1}\right] \ldots \operatorname{Pr}\left[y_{k-l}\right]\right) \\
= & \sum_{t}\left(\sum_{z_{1}+\ldots+z_{l}=t} \operatorname{Pr}\left[z_{1}\right] \ldots \operatorname{Pr}\left[z_{l}\right]\right) \\
& \times\left(\sum_{x_{1}+\ldots+x_{k-l}=n-t} \operatorname{Pr}\left[x_{1}\right] \ldots \operatorname{Pr}\left[x_{k-l}\right]\right) \\
& \times\left(\sum_{y_{1}+\ldots+y_{k-l}=m-t} \operatorname{Pr}\left[y_{1}\right] \ldots \operatorname{Pr}\left[y_{k-l}\right]\right) \\
= & \sum_{t} \mu_{l}(t) \mu_{k-l}(n-t) \mu_{k-l}(m-t)=\Delta \quad \text { (say). }
\end{aligned}
$$


We are going to show that $\Delta \leq n^{-l /(2 k)+o(1)}$ by making use of the following estimates for $\mu_{l}(n)$ from [7] (Lemma 8, p. 252):

$$
\mu_{l}(n) \leq n^{-1+l / k+o(1)} \quad \text { for } 2 \leq l \leq k-1 .
$$

For technical reasons, fix $\varepsilon=l /(4 k)$. Now pick $t_{0}$ such that

$$
\mu_{l}(t) \leq t^{-1+l / k+\varepsilon} \quad \text { for } t>t_{0} .
$$

The proof that $\Delta \leq n^{-l /(2 k)+o(1)}$ gets quite technical, and can be omitted on the first reading without loss of understanding of the rest of the paper.

Case 1. Let us assume that $m=O\left(n^{\delta}\right)$ for $\delta<1$.

Case $1(\mathrm{a}) . t \leq t_{0}$ :

$$
\begin{aligned}
\Delta_{1} & =\sum_{t \leq t_{0}}\left(\mu_{l}(t) \mu_{k-l}(n-t) \mu_{k-l}(m-t)\right) \\
& <n^{-1+(k-l) / k+o(1)} m^{-1+(k-l) / k+o(1)} \sum_{t \leq t_{0}} H \\
& <n^{-1+(k-l) / k+o(1)}=n^{-l / k+o(1)} .
\end{aligned}
$$

Case 1(b). $m-t_{0}<t \leq m$ :

$$
\begin{aligned}
\Delta_{2} & =\sum_{m-t_{0}<t \leq m}\left(\mu_{l}(t) \mu_{k-l}(n-t) \mu_{k-l}(m-t)\right) \\
& <\left(m^{-1+l / k+o(1)} n^{-1+(k-l) / k+o(1)}\right) \sum_{m-t_{0}<t \leq m} \mu_{k-l}(m-t) \\
& <\left(n^{-1+(k-l) / k+o(1)}\right) \sum_{m-t_{0}<t \leq m} H \\
& <\left(n^{-1+(k-l) / k+o(1)}\right)=n^{-l / k+o(1)} .
\end{aligned}
$$

Case $1(\mathrm{c}) \cdot t_{0}<t \leq m-t_{0}$ :

$$
\begin{aligned}
\Delta_{3} & =\sum_{t_{0}<t \leq m-t_{0}}\left(\mu_{l}(t) \mu_{k-l}(n-t) \mu_{k-l}(m-t)\right) \\
& <\left(n^{-1+(k-l) / k+o(1)} m^{-1+(k-l) / k+o(1)}\right) \sum_{t_{0}<t \leq m-t_{0}} t^{-1+l / k+\varepsilon} .
\end{aligned}
$$

We can now estimate the sum by an integral over the full range $0 \leq t \leq m$ :

$$
\begin{aligned}
\Delta_{3} & <\left(n^{-1+(k-l) / k+o(1)} m^{-1+(k-l) / k+o(1)}\right)\left(\int_{0}^{m} t^{-1+l / k+\varepsilon}+O(1)\right) \\
& =\left(n^{-1+(k-l) / k+o(1)} m^{-1+(k-l) / k+o(1)}\right)\left(m^{l / k+\varepsilon}+O(1)\right) \\
& <n^{-1+(k-l) / k+o(1)}=n^{-l / k+o(1)} .
\end{aligned}
$$


C a se 2. In this case, we let $m=\Theta(n)$.

Case $2(\mathrm{a}) . t \leq t_{0}$ :

$$
\begin{aligned}
\Delta_{1}^{\prime} & =\sum_{t \leq t_{0}}\left(\mu_{l}(t) \mu_{k-l}(n-t) \mu_{k-l}(m-t)\right) \\
& <n^{-1+(k-l) / k+o(1)} m^{-1+(k-l) / k+o(1)} \sum_{t \leq t_{0}} H \\
& <n^{-1+(k-l) / k+o(1)} m^{-1+(k-l) / k+o(1)} \\
& <n^{-2+2(k-l) / k+o(1)}, \quad \text { since } m=\Theta(n) \\
& =n^{-l / k+o(1)}
\end{aligned}
$$

Case 2(b). $m-t_{0}<t \leq m$ :

$$
\begin{aligned}
\Delta_{2}^{\prime} & =\sum_{m-t_{0}<t \leq m}\left(\mu_{l}(t) \mu_{k-l}(n-t) \mu_{k-l}(m-t)\right) \\
& <\left(m^{-1+l / k+o(1)}\right) \sum_{m-t_{0}<t \leq m}(n-t)^{-1+(k-l) / k+o(1)} H \\
& =\left(m^{-1+l / k+o(1)}\right) \sum_{m-t_{0}<t \leq m}(n-t)^{-1+(k-l) / k+o(1)} \\
& <\left(m^{-1+l / k+o(1)}\right)\left(t_{0} \times(n-m)^{-1+(k-l) / k+o(1)}\right) \\
& =\left(m^{-1+l / k+o(1)}\right)\left(n^{-1+(k-l) / k+o(1)}\right) \\
& =\left(n^{-1+l / k+o(1)}\right)\left(n^{-l / k+o(1)}\right) \\
& <n^{-1+o(1)} .
\end{aligned}
$$

Case $2(\mathrm{c}) . t_{0}<t \leq m / 2$ :

$$
\begin{aligned}
\Delta_{3}^{\prime} & =\sum_{t_{0} \leq t \leq m / 2}\left(\mu_{l}(t) \mu_{k-l}(n-t) \mu_{k-l}(m-t)\right) \\
& <\left(n^{-1+(k-l) / k+o(1)} m^{-1+(k-l) / k+o(1)}\right) \sum_{t_{0} \leq t \leq m / 2} t^{-1+l / k+\varepsilon} .
\end{aligned}
$$

We can now estimate the sum by an integral over the full range $0 \leq t \leq m$ :

$$
\begin{aligned}
\Delta_{3}^{\prime} & <\left(n^{-1+(k-l) / k+o(1)}\right)\left(m^{-1+(k-l) / k+o(1)}\right)\left(\int_{0}^{m} t^{-1+l / k+\varepsilon}+O(1)\right) \\
& =\left(n^{-1+(k-l) / k+o(1)}\right)\left(m^{-1+(k-l) / k+o(1)}\right)\left(m^{l / k+\varepsilon}+O(1)\right) \\
& =\left(n^{-l / k+o(1)} m^{\varepsilon+o(1)}\right) \\
& <n^{-l / k+\varepsilon+o(1)}=n^{-(3 l) /(4 k)+o(1)} .
\end{aligned}
$$


Case $2(\mathrm{~d}) \cdot m / 2<t \leq m-t_{0}$ :

$$
\begin{aligned}
\Delta_{4}^{\prime} & =\sum_{m / 2<t \leq m-t_{0}}\left(\mu_{l}(t) \mu_{k-l}(n-t) \mu_{k-l}(m-t)\right) \\
& <\left(m^{-1+l / k+o(1)}\right) \sum_{m / 2<t \leq m-t_{0}}(n-t)^{-1+(k-l) / k+\varepsilon}(m-t)^{-1+(k-l) / k+\varepsilon} \\
& <\left(m^{-1+l / k+o(1)}\right) \sum_{m / 2<t \leq m-t_{0}}(m-t)^{-2 l / k+2 \varepsilon} .
\end{aligned}
$$

Once again, we estimate the sum by an integral over the full range $0 \leq t \leq m$ :

$$
\begin{aligned}
\Delta_{4}^{\prime} & <m^{-1+l / k+o(1)}\left(\int_{0}^{m}(m-t)^{-2 l / k+2 \varepsilon}+O(1)\right) \\
& <m^{-1+l / k+o(1)}\left(-\left.(m-t)^{1-2 l / k+2 \varepsilon}\right|_{0} ^{m}+O(1)\right) \\
& =m^{-l / k+2 \varepsilon+o(1)} \\
& =n^{-l / k+2 \varepsilon+o(1)}=n^{-l /(2 k)+o(1)} .
\end{aligned}
$$

From Cases 1 and 2, we can conclude that

$$
E\left[R_{l}(m, n)\right] \leq n^{-l /(2 k)+o(1)} .
$$

This implies

$$
E[R(m, n)]=\sum_{l=1}^{k-1} E\left[R_{l}(m, n)\right] \leq n^{-l /(2 k)+o(1)} .
$$

Lemma 5. (i) $\operatorname{Pr}\left[R^{*}(m, n)>8 k\right]<n^{-4+o(1)}$.

(ii) a.a. $\exists c^{*}$ such that $R^{*}(m, n)<c^{*}$ for all $m<n$.

Proof. (i) We use the disjointness lemma from [7] to prove the first part; thus

$$
\begin{aligned}
\operatorname{Pr}\left[R^{*}(m, n)>8 k\right] & <\frac{(E[R(m, n)])^{8 k}}{(8 k) !} \\
& <\frac{1}{(8 k) !}\left(n^{-l /(2 k)+o(1)}\right)^{8 k} \\
& =n^{-4 l+o(1)}<n^{-4+o(1)}, \quad \text { since } l \geq 1 .
\end{aligned}
$$

(ii) Let $A_{m n}$ denote the event that $R^{*}(m, n)>8 k$. Then the first part of this lemma implies that $\operatorname{Pr}\left[A_{m n}\right]<n^{-4+o(1)}$. There are at most $n^{2}$ pairs $(m, n)$ such that $m<n$, and since $n^{2} \operatorname{Pr}[A(m, n)]<\infty$, by the Borel-Cantelli lemma (see e.g. [7]), this implies that

a.a. $\exists n^{*}$ such that $R^{*}(m, n)<8 k$ for all $m<n$, whenever $n>n^{*}$. 
But for any finite $n^{*}, R^{*}\left(m, n^{*}\right)$ is certainly bounded for all $m<n^{*}$. Thus we conclude that

a.a. $\exists c^{*}$ such that $R^{*}(m, n)<c^{*}$ for all $m<n$.

Proof of Lemma 3 . Let us define the following equivalence relation "०" on the numbers in $\left|S_{\mathcal{S}}(m) \cap S_{\mathcal{S}}(n)\right|: x \circ y$ iff

$$
x+a_{1}+\ldots+a_{k-1}=m \text { and } y+a_{1}^{\prime}+\ldots+a_{k-1}^{\prime}=n
$$

and moreover,

$$
\left\{a_{1}, \ldots, a_{k-1}\right\} \cap\left\{a_{1}^{\prime}, \ldots, a_{k-1}^{\prime}\right\} \neq \emptyset .
$$

(Thus $x$ and $y$ are related iff $x$ and $y$ belong to some overlapping pair of representations for $m$ and $n$.) The number of equivalence classes defined by ○ is bounded since $R^{*}(m, n)$ is bounded. Moreover, for each $x \in \operatorname{class} C_{x}$, there are at most a bounded number of $y \in C_{x}$, since both $r_{\mathcal{S}}^{\prime}(m ; a)$ and $r_{\mathcal{S}}^{\prime}(n ; a)$ are bounded. Thus each equivalence class is also bounded, and hence $\left|S_{\mathcal{S}}^{\prime}(m) \cap S_{\mathcal{S}}^{\prime}(n)\right|$ is bounded.

4. Conclusions. Theorems 1 and 2 along with Lemmas $1-3$ imply that the asymptotic basis constructed in [7] contains a minimal (strict) and an $\aleph_{0}$-minimal (strict) asymptotic basis.

Erdős and Nathanson [3] obtained the following very simple criterion for an asymptotic basis $A$ of order 2 to contain a minimal asymptotic basis.

THEOREM $4(\mathrm{EN})$. If there exists a constant $c>1 / \log \left(\frac{4}{3}\right)$, such that $r_{A}^{\prime}(n)>c \log n$ for all sufficiently large $n$, then A contains a minimal asymptotic basis of order 2 .

The combinatorial lemma at the heart of this theorem has since been generalized by Jia [9] and Nathanson [10]. However, the search for an analogue of Theorem [EN] remains open for bases of order $k>2$. Clearly, this question requires some new ideas.

Another very interesting problem, which is open even for bases of order 2 , is if the weaker condition that $r_{A}^{\prime}(n) \rightarrow \infty$ is sufficient to imply that $A$ must contain a minimal asymptotic basis. Perhaps this conjecture is too optimistic, but it is possible that $r_{A}^{\prime}(n)>c \log n$, for every $c>0$, is sufficient to imply that $A$ must contain a minimal asymptotic basis.

\section{References}

[1] P. Erdös and M. B. Nathanson, Oscillations of bases for the natural numbers, Proc. Amer. Math. Soc. 53 (1975), 253-258.

[2] —,-, Independence of solution sets in additive number theory, in: Probability, Statistical Mechanics, and Number Theory, G.-C. Rota (ed.), Adv. Math. Suppl. Stud. 9 (1986), 97-105. 
[3] P. Erdős and M. B. Nathanson, Systems of distinct representatives and minimal bases in additive number theory, in: Number Theory, Carbondale 1979, M. B. Nathanson (ed.), Lecture Notes in Math. 751, Springer, Heidelberg, 1979, 89-107.

[4] - - - Problems and results on minimal bases in additive number theory, in: Number Theory, New York 1985-86, D. V. Chudnovsky, G. V. Chudnovsky, H. Cohn, and M. B. Nathanson (eds.), Lecture Notes in Math. 1240, Springer, Heidelberg, 1987, $87-96$.

[5] P. Erdös and R. Rado, Intersection theorems for systems of sets, J. London Math. Soc. 35 (1960), 85-90.

[6] P. Erdős and A. Rényi, Additive properties of random sequences of positive integers, Acta Arith. 6 (1960), 83-110.

[7] P. Erdős and P. Tetali, Representations of integers as the sum of $k$ terms, Random Structures and Algorithms 1 (1990), 245-261.

[8] H. Halberstam and K. F. Roth, Sequences, Springer, Heidelberg, 1983.

[9] X.-D. Jia, Simultaneous systems of representatives for finite families of finite sets, Proc. Amer. Math. Soc. 104 (1988), 33-36.

[10] M. B. Nathanson, Simultaneous systems of representatives for families of finite sets, Proc. Amer. Math. Soc. 103 (1988), 1322-1326.

MATHEMATICAL INSTITUTE

HUNGARIAN ACADEMY OF SCIENCES

REÁLTANODA U. 13-15

H-1053 BUDAPEST

HUNGARY

DEPARTMENT OF MATHEMATICS

LEHMAN COLLEGE (CUNY)

BRONX, NEW YORK 10468

U.S.A.
AT\&T BELL LABORATORIES

MURRAY HILL

NEW JERSEY 07974

U.S.A.

E-mail: PRASAD@RESEARCH.ATT.COM 